\title{
Editorial
}

\section{LOFAR Surveys: a new window on the Universe}

The LOFAR telescope, one of the low frequency precursors of SKA, is being used to survey the full northern celestial hemisphere in the $120 \mathrm{MHz}$ band with unprecedented resolution and sensitivity. This special issue of Astronomy \& Astrophysics presents the first data release of the LOFAR Two-metre Sky Survey (LOTSS), along with selected early results. The newly released mosaicked images cover 424 square degrees in a single high galactic latitude field with $6^{\prime \prime}$ resolution. The release includes an extracted catalog of 326000 sources, complete to $0.35 \mathrm{mJy}$ for point sources. $70 \%$ of these sources are associated with an optical counterpart, and photometric redshifts are provided for those. The early results of the survey span a wide range of mostly extragalactic topics, from the physics of active galactic nuclei to the intergalactic magnetic field, through interstellar medium and clusters of galaxies. This represents only a small sample of what is yet to come, but already hints at the great potential of the full survey.

Françoise Combes, Andrea Ferrara, Thierry Forveille, Rubina Kotak, Steve Shore, and Mario Tafalla Astronomy \& Astrophysics Editors 